\title{
November 2014 Imaging Case of the Month
}

\author{
Michael B. Gotway, MD \\ Department of Radiology \\ Mayo Clinic Arizona \\ Scottsdale, AZ
}

Clinical History: A 38-year-old non-smoking woman presented with complaints of intermittent dry cough, occasional vomiting, and dyspnea, occasionally with fever and chills. She indicated that she has suffered recurrent bouts of bronchitis and pneumonia annually over the previous 10 years. The patient had a history of upper arm localized melanoma resection 10 years earlier. She had smoked for 10 years, but quit one year prior to presentation. Her past medical and surgical histories were otherwise unremarkable.

Frontal and lateral chest radiography (Figure 1) was performed.

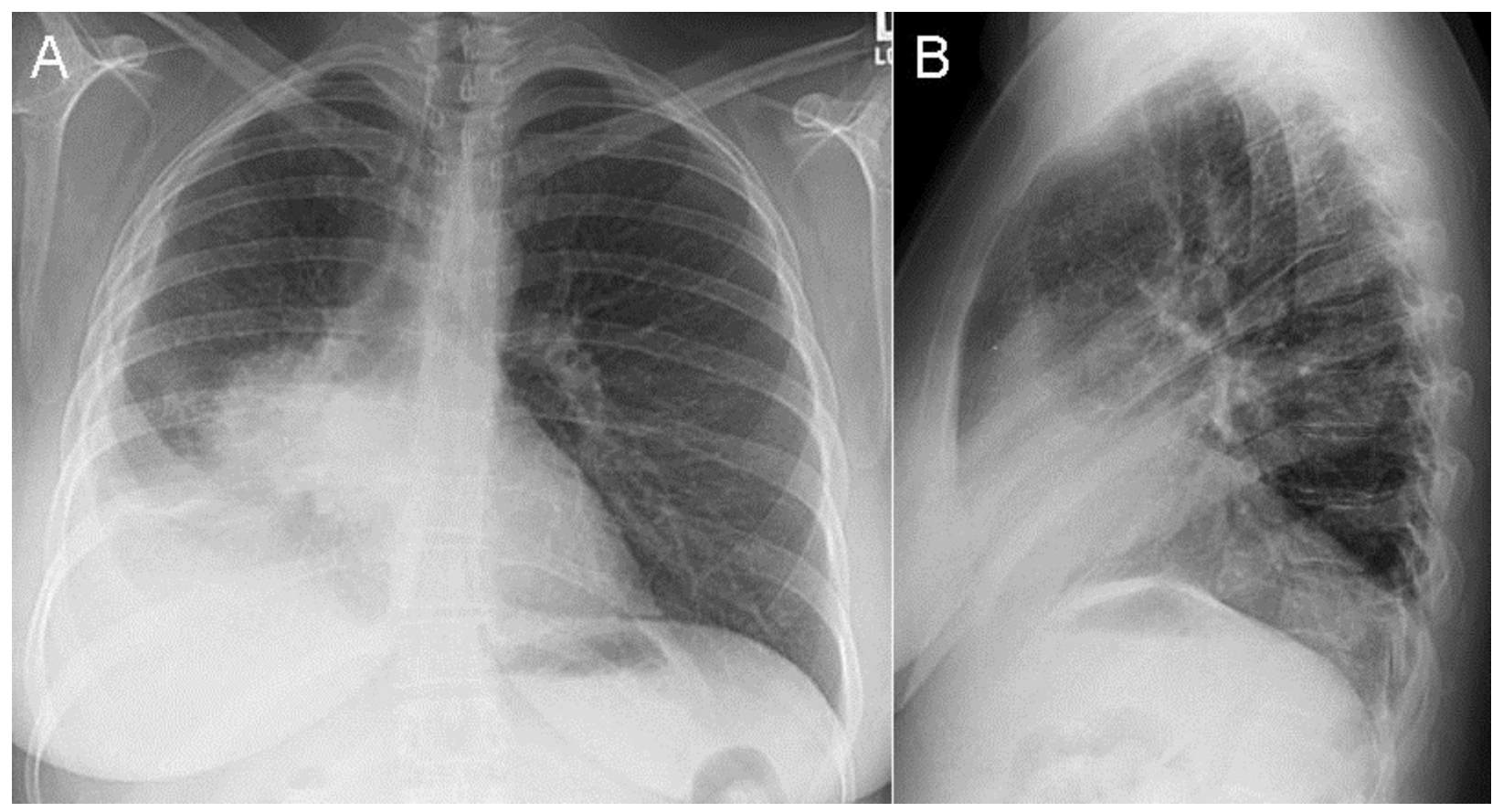

Figure 1. Frontal (A) and lateral (B) chest radiography. 
Which of the following statements regarding the chest radiograph is most accurate?

1. The chest radiograph shows asymmetric pulmonary vascularity

2. The chest radiograph shows bilateral linear and reticular opacities and diminished lung volumes suggesting fibrotic lung disease

3. The chest radiograph shows mild, bilateral, streaky central opacities, possibly reflecting airway thickening

4. The chest radiograph shows numerous small nodules

5. The chest radiograph shows volume loss involving the right thorax with a masslike opacity projected over the right hilum 


\section{Correct!}

5. The chest radiograph shows volume loss involving the right thorax with a mass-like opacity projected over the right hilum

The chest radiograph shows a rounded opacity projected over and partially obscuring the right hilum. There is significant volume loss in the right thorax- note shift of the trachea towards the right and elevation of the right diaphragm. A right pleural effusion is also present. The left lung is clear. There is no evidence of cystic or fibrotic lung disease, and the visible central airways do not show thickening. There are no "streaky central opacities on the left side. There is no evidence of a small nodular pattern.

Which of the following represents the next, most appropriate step for the assessment of the findings at chest radiography?

1. ${ }^{18}$ FDG-PET scan

2. Contrast-enhanced thoracic CT

3. Repeat chest radiography

4. Thoracic MRI

5. Ventilation - perfusion scintigraphy 


\section{Correct!}

\section{Contrast-enhanced thoracic CT}

Contrast-enhanced thoracic CT is the next most appropriate step for the assessment of this patient. While thoracic MRI and ${ }^{18}$ FDG-PET scanning are both reasonable considerations, contrast -enhanced CT will provide the best assessment for the anatomic extent of disease involving the right pleural space, hila, mediastinum, central airways (given the right lung volume loss) and lung parenchyma. Ventilation-perfusion scintigraphy may provide some useful information regarding relative lung perfusion, but cannot provide a detailed assessment of the anatomic disease distribution and extent for this patient. Given the extensive abnormalities at chest radiography and the patient's symptoms, an active investigation, as opposed to a conservative surveillance posture, is warranted.

The patient underwent contrast-enhanced thoracic CT (Figure 2) was performed.

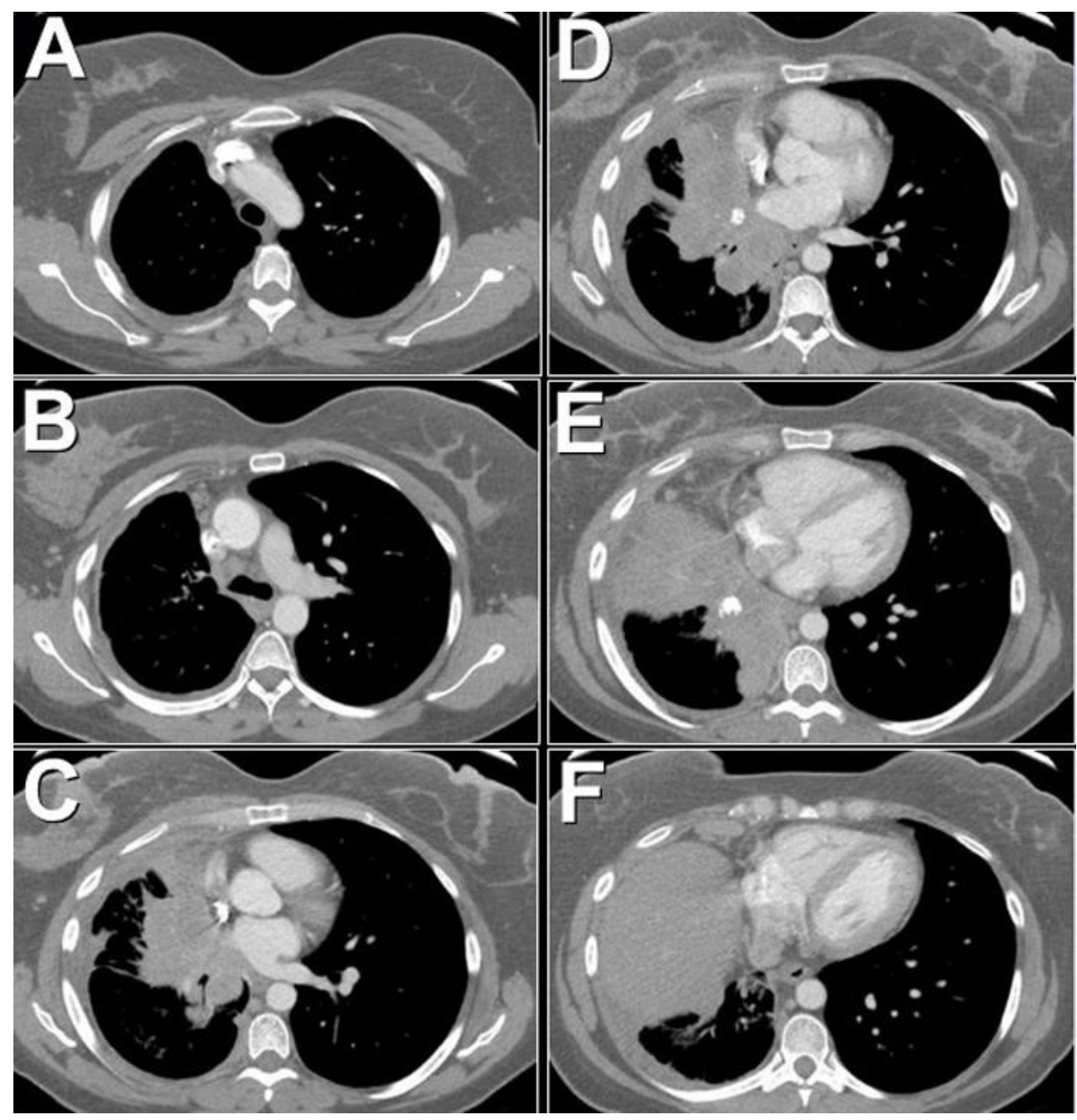

Figure 2. Representative images from the contrast-enhanced thoracic CT in soft tissue windows. 
Which of the following statements regarding this study is most accurate?

1. The thoracic CT shows a large anterior mediastinal mass

2. The thoracic CT shows a multilobulated right pleural space mass with pleural liquid

3. The thoracic CT shows a right chest wall mass invading the right pleural space

4. The thoracic CT shows a right-sided perihilar soft tissue mass associated with mediastinal lymph node enlargement and right pleural thickening

5. The thoracic CT shows bilateral peribronchial and mediastinal lymph node enlargement with low attenuation necrosis 


\section{Correct!}

4. The thoracic CT shows a right-sided perihilar soft tissue mass associated with mediastinal lymph node enlargement and right pleural thickening

Thoracic CT shows a large right-sided mass with extensive mediastinal contact and compression of the right pulmonary arteries and veins. Multifocal mediastinal lymph node enlargement is also evident. The right paramediastinal mass shows foci of calcification (Figure 3).

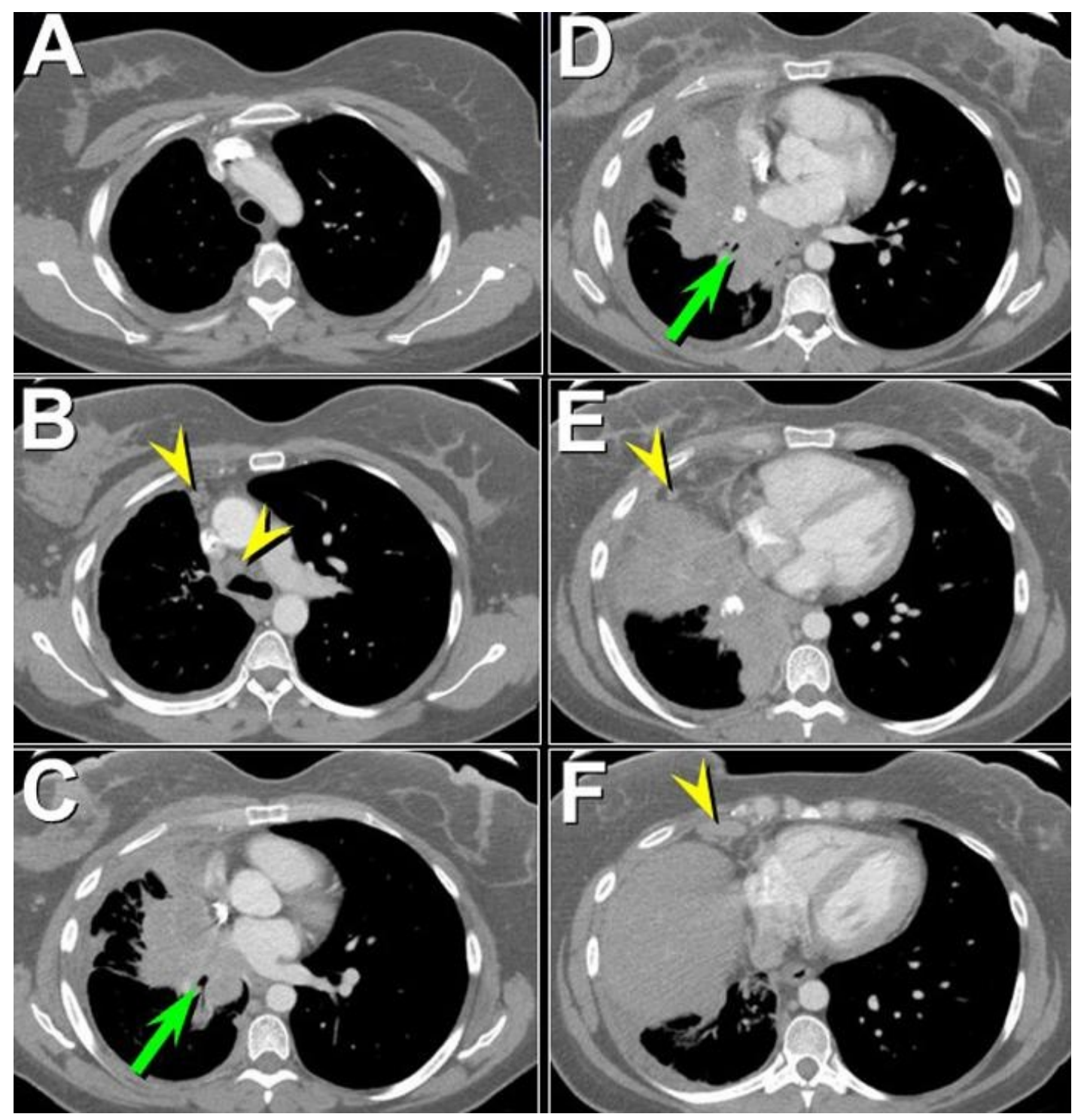

Figure 3. Contrast-enhanced thoracic CT shows a large right-sided mass with extensive mediastinal contact and compression of the right pulmonary arteries and veins. Multifocal mediastinal lymph node enlargement (arrowheads, B, E, and F) is also evident. The right paramediastinal mass shows foci of calcification. The mass compresses and narrows the right lower lobe bronchus (arrows, $\mathbf{C}$ and $\mathbf{D}$ ), resulting in the right lung volume loss noted at chest radiography. 
The mediastinal lymph node enlargement is unilateral, not bilateral. While circumferential pleural thickening is present, the findings should not be described as a "multilobulated pleural space mass", and no pleural liquid is present. No dominant anterior mediastinal mass is present. No evidence of a chest wall mass is seen.

Clinical course: The patient subsequently underwent 18FDG-PET scanning (Figure 4) for further evaluation of the findings at thoracic CT.

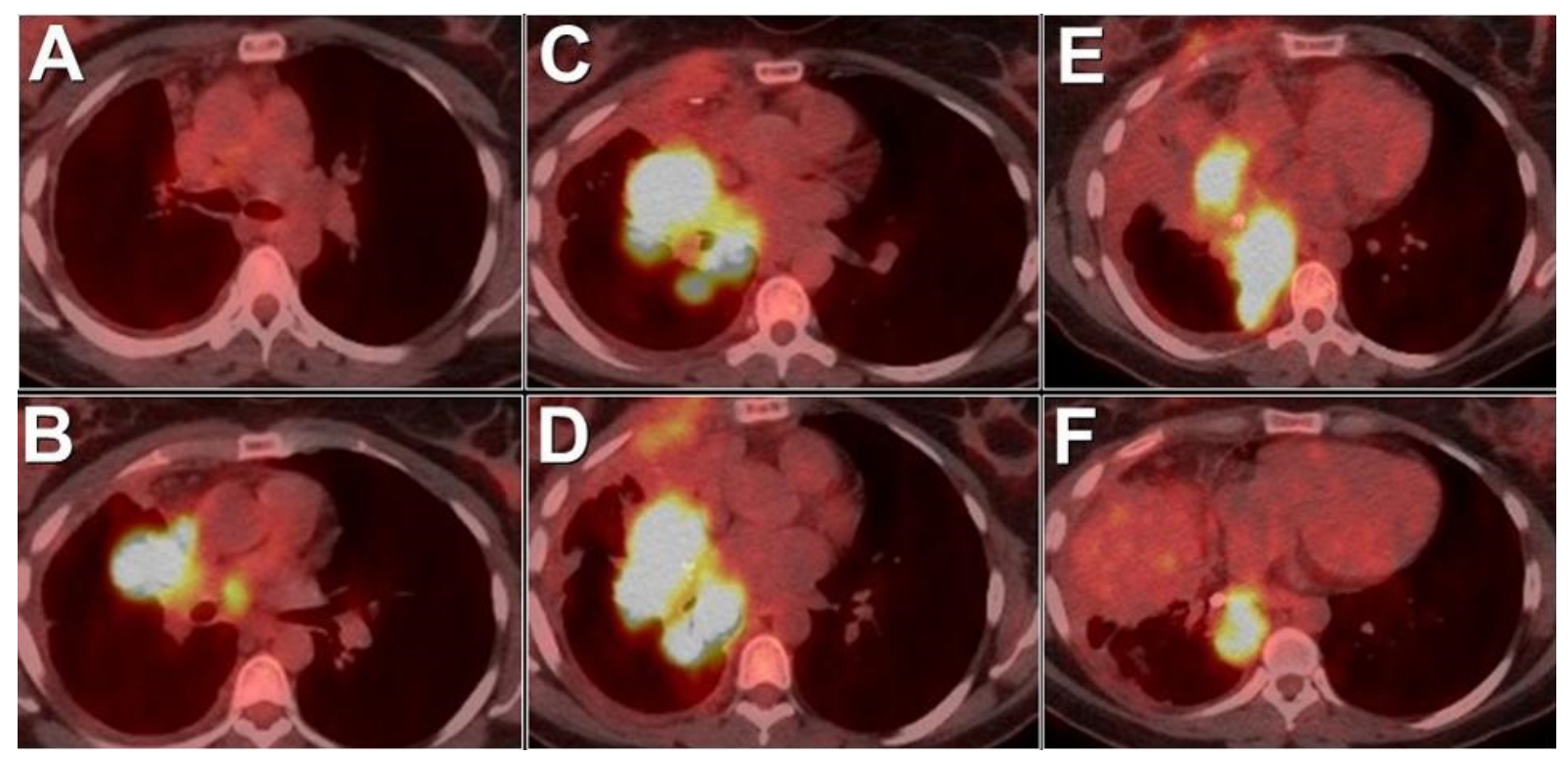

Figure 4. 18FDG-PET scan.

Which of the following statements regarding this imaging study is most accurate?

1. The 18FDG-PET scan is degraded by artifact and not readily interpretable

2. The 18FDG-PET shows hypermetabolic activity within the enlarged mediastinal nodes seen at thoracic $\mathrm{CT}$, but not within the paramediastinal lesion

3. The 18FDG-PET shows hypermetabolism in the paramediastinal lesion but not within any of the enlarged mediastinal nodes seen at thoracic CT

4. The 18FDG-PET shows hypermetabolism in the paramediastinal lesion and several of the enlarged mediastinal lymph nodes seen at thoracic CT

5. The 18FDG-PET shows no significant metabolic activity in the thorax 


\section{Correct!}

\section{The 18FDG-PET shows hypermetabolism in the paramediastinal lesion and several of the enlarged mediastinal lymph nodes seen at thoracic CT}

The ${ }^{18}$ FDG-PET scan is of good quality and readily interpretable. The study shows extensive hypermetabolism in the right paramediastinal mass as well as several, but not all, of the enlarged mediastinal lymph nodes seen at thoracic CT (Figure 5).

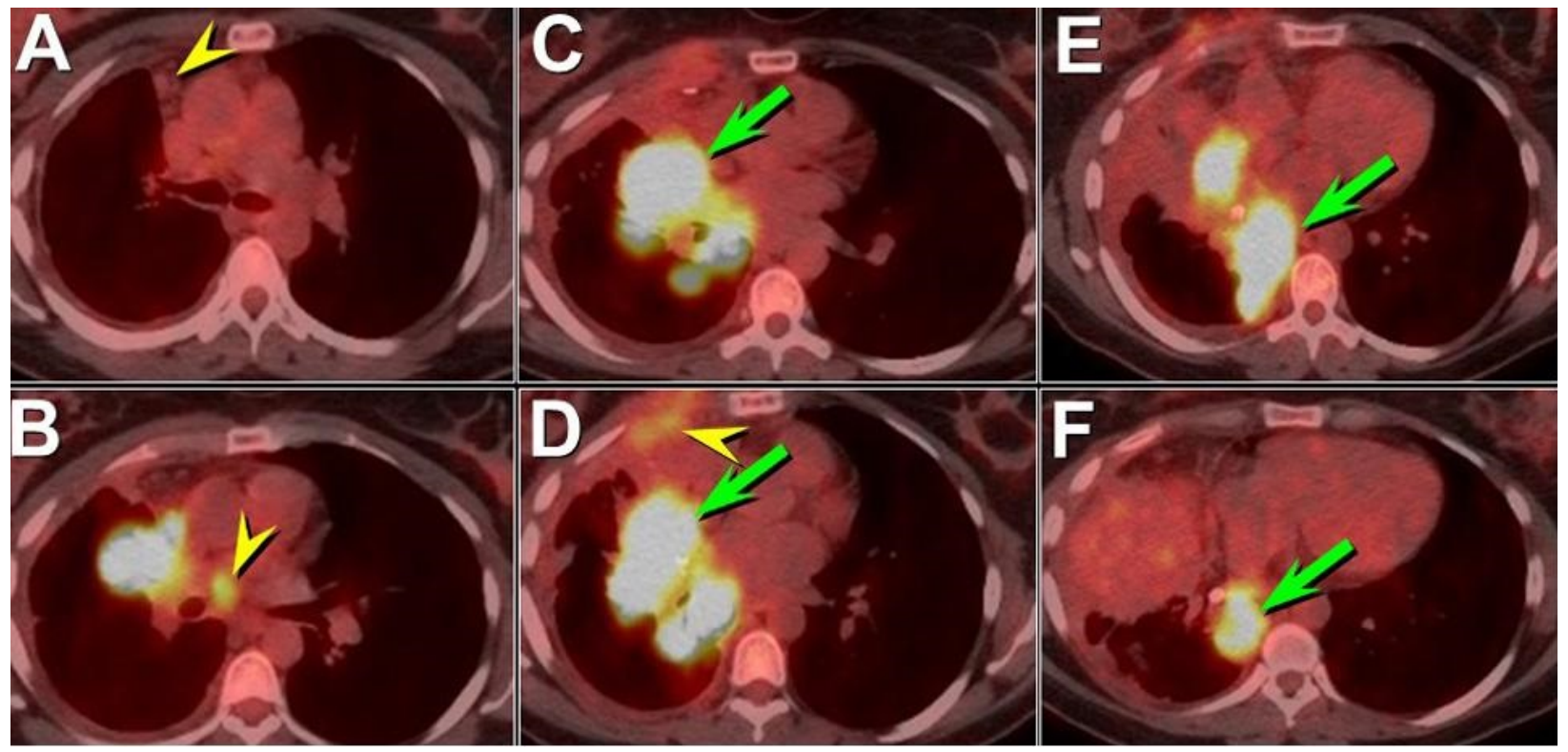

Figure 5. ${ }^{18}$ FDG-PET shows extensive hypermetabolism in the right paramediastinal mass (arrows, $\mathbf{C}-\mathbf{F}$ ) as well as within several of the enlarged mediastinal lymph nodes (arrowheads, B and D) seen at thoracic CT (one right internal mammary lymph nodearrowhead in A- shows little metabolic activity.

These findings suggest the presence of malignancy, but are not specific; a number of infectious and non-infectious inflammatory lesions may show elevated glucose metabolism at ${ }^{18}$ FDG-PET scanning.

Given the contrast-enhanced thoracic CT and 18FDG-PET scan findings, which of the following is the next most appropriate step for obtaining a diagnosis in this patient?

1. Bronchoscopy with transbronchial biopsy and endobronchial ultrasound

2. Mini-thoracotomy

3. Percutaneous transthoracic fine needle aspiration and core biopsy

4. Thoracoscopic surgical lung biopsy

5. Any of the above 


\section{Correct! \\ 5. Any of the above}

There are a number of "targets" that would be suitable for obtaining a tissue diagnosis in this patient. Percutaneous transthoracic fine needle aspiration and core biopsy could access the right paramediastinal mass, whereas the compression and narrowing of the right lower lobe bronchus, as well as the mediastinal lymph node enlargement [especially in the right paratracheal, peribronchial, and subcarinal stations] make bronchoscopy with endobronchial ultrasound and transbronchial biopsy a potentially useful procedure in this patient. Both of these methods are minimally invasive, and have the potential advantage of providing a tissue diagnosis with less expense and potential morbidity than the more invasive surgical techniques of thoracoscopic lung biopsy and a mini-thoracotomy; however, these latter procedures have the advantage of obtaining larger tissue samples for establishing a definitive diagnosis.

Further clinical course: The patient subsequently underwent bronchoscopy (Figure 6), but the tissue obtained was non diagnostic.

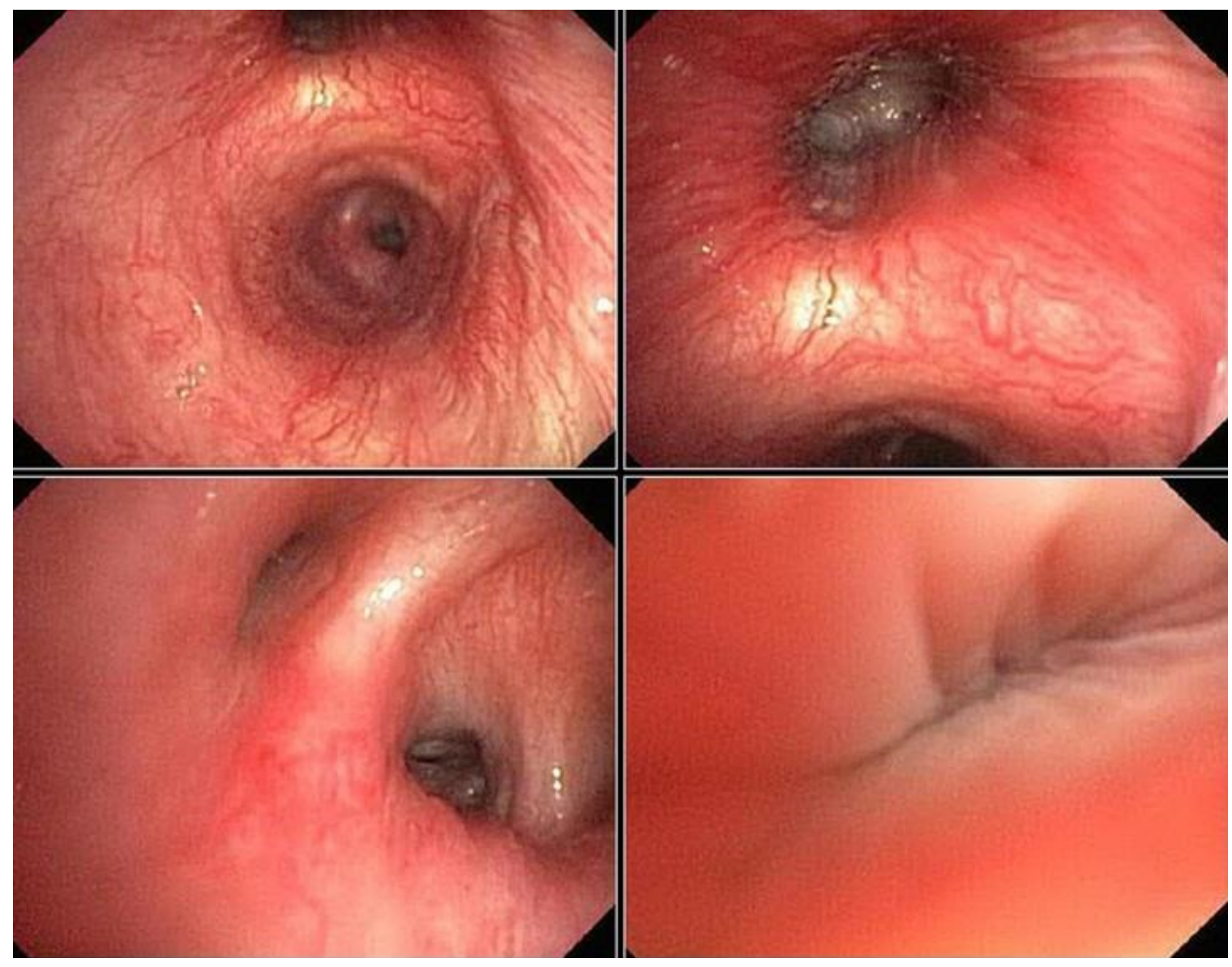

Figure 6. Bronchoscopy shows few intrinsic airway abnormalities: some erythema around the orifice of the right upper lobe bronchus is present, and extrinsic compression of the right middle lobe bronchus is seen. 
Lymph node stations $4 \mathrm{R}, 7$, and $11 \mathrm{R}$ were samples, and mucosal biopsies of the right upper, middle, and lower lobes were performed. Extrinsic compression on the right middle lobe bronchus was noted, as was some erythema near the orifice of the right upper lobe bronchus.

The patient then underwent repeat chest radiography (Figure 7) repeat thoracic CT (Figure 8).

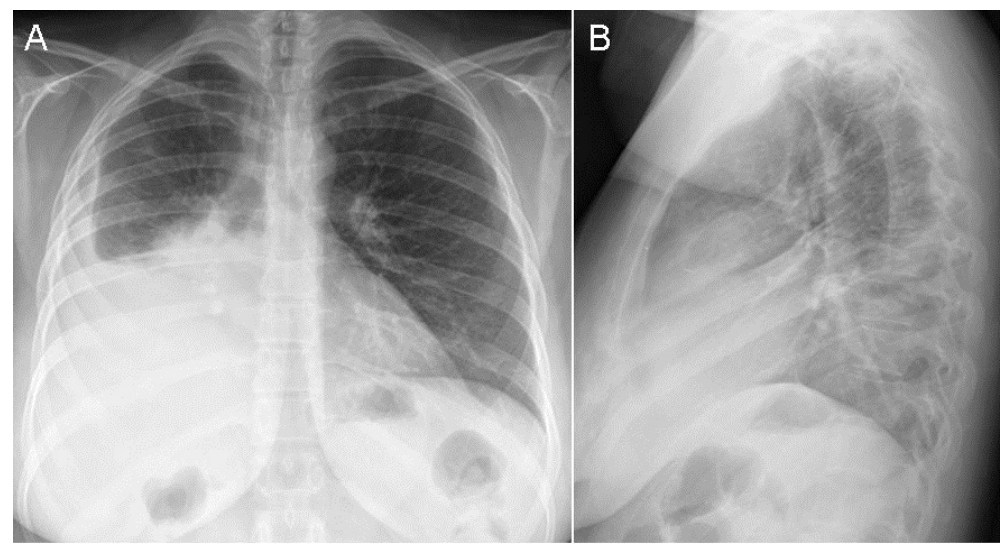

Figure 7. Frontal (A) and lateral (B) chest radiography performed 2 months following presentation imaging (Figure 1).

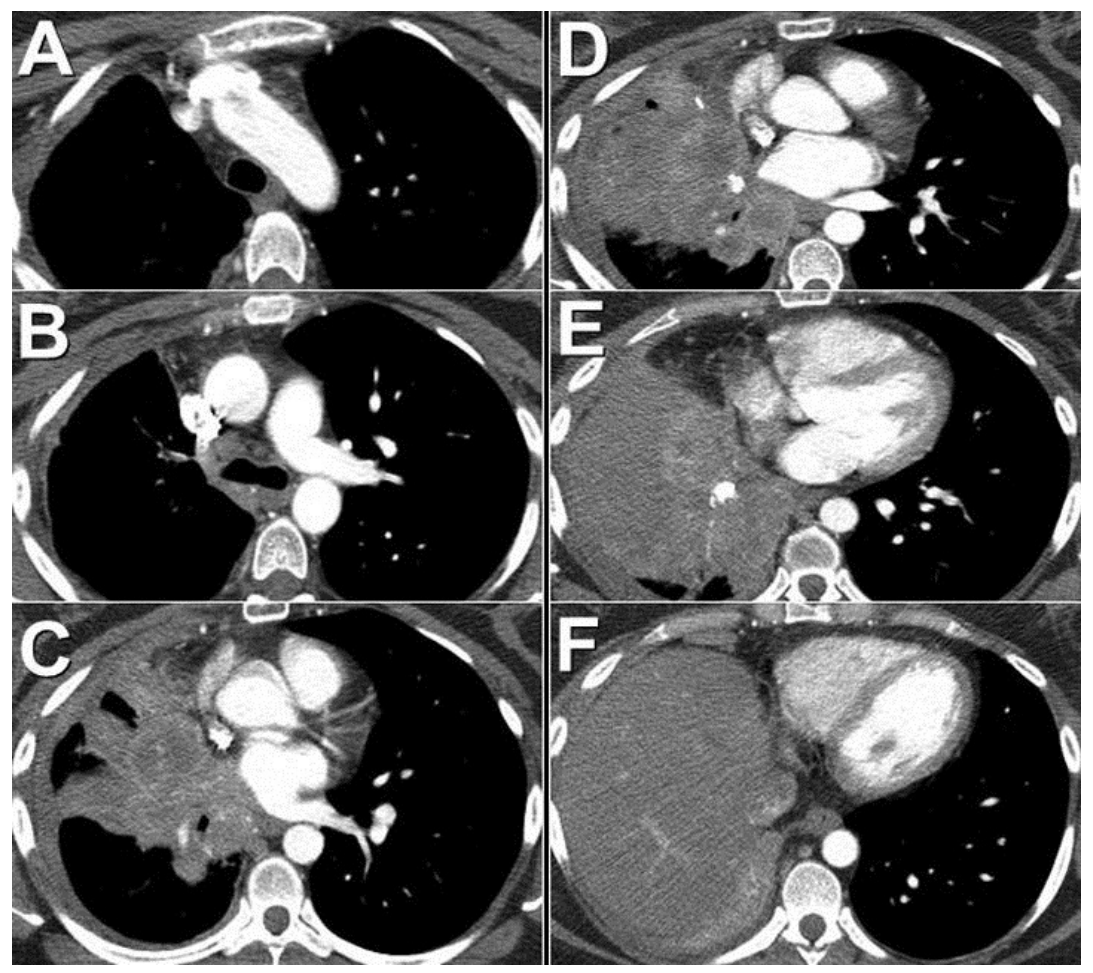

Figure 8. Contrast-enhanced thoracic CT in soft tissue windows performed 2 months following presentation imaging (Figure 2). 
Which of the following statements regarding these imaging studies is most accurate?

1. The chest radiograph and thoracic $\mathrm{CT}$ both new left mediastinal lymph node enlargement and new left pleural liquid

2. The chest radiograph and thoracic CT both show regression in the previously noted mediastinal lymph node enlargement, right pleural abnormalities, and right paramediastinal mass

3. The chest radiograph and thoracic $\mathrm{CT}$ both show slight progression in the previously noted abnormalities

4. The thoracic CT shows a new anterior mediastinal mass

5. The thoracic CT shows a new, large pericardial effusion 


\section{Correct! \\ 3. The chest radiograph and thoracic $C T$ both show slight progression in the previously noted abnormalities}

The chest radiograph shows relatively more right basal opacity with greater blunting of the right costophrenic angle, suggesting slight worsening in the previously noted abnormalities. The repeat thoracic CT appears fairly similar to the thoracic CT performed at presentation (Figure 2), perhaps with slight increase in size of the right paramediastinal process and associated consolidation. There is no evidence of left pleural liquid or new left-sided mediastinal lymph node enlargement, nor is there a new anterior mediastinal mass. The abnormalities noted on imaging at presentation have not shown any regression. No pericardial effusion is present.

Given the available data to this point, which of the following is the next most appropriate step for obtaining a diagnosis in this patient?

1. Percutaneous transthoracic fine needle aspiration and core biopsy

2. Repeat bronchoscopy with endobronchial ultrasound and transbronchial biopsy

3. Thoracoscopic surgical lung biopsy

4. 1 or 3

5. None of the above 


\section{Correct!}

\section{1 or 3}

Given the lack of a tissue diagnosis from the previously performed bronchoscopic biopsy, a procedure capable of obtaining a larger tissue sample is now indicated. Therefore, repeat bronchoscopy with endoscopic ultrasound and transbronchial biopsy is not the best choice among those listed. Both percutaneous transthoracic fine needle aspiration and core biopsy and surgical biopsy procedures provide larger tissue samples than bronchoscopic biopsies [albeit at a higher rate of associated complications]. The surgical approach to a tissue diagnosis- either mediastinoscopy or video-assisted thoracoscopic biopsy- could target the right paratracheal lymph nodes or pleural space abnormalities in this patient. Percutaneous transthoracic fine needle aspiration and core biopsy could target the low attenuation areas in the medial right middle lobe and / or lower lobe (Figure 8).

Further clinical course: The patient underwent percutaneous transthoracic fine needle aspiration and core biopsy, but the tissue obtained at this procedure did not yield a diagnosis. The patient subsequently underwent a thoracoscopic surgical biopsy procedure, but that procedure was complicated by adhesions, and thus the procedure was converted to a mini-thoracotomy. The right pleural abnormality was sampled, as were portions of the right paramediastinal mass. The surgical lung biopsy material showed Fibroinflammatory process involving lung and mediastinum characterized by fibrous and myofibroblastic proliferation, sclerotic nodules, streaming fibrosis, polyclonal plasma cells, mixed T and B lymphocytic infiltration and increased vascularity and entrapped lung, but with no features of malignancy. The inflammatory infiltrate consisted of lymphocytes and plasma cells with scattered eosinophils, and in some areas, the fibrosis appeared quite dense and suggestive of a keloidal type deposition. Immunohistochemistry studies showed only rare scattered B-cells with normal coexpression of PAX5, and majority of the lymphocytes were small T-cells. No ReedSternberg cells were identified with the aid of CD30, CD15 stains. EMA and PC3 stains highlighted the largely decoyed pulmonary parenchyma in the background. Calretinin and CK5 stains were negative. In situ hybridization for kappa and lambda light chain highlighted a polytypic plasma cell population with only a small number of IgG positive plasma cells demonstrating low co-expression of IgG4. Gram staining was negative.

Given these characteristics, which of the following represents the most likely diagnosis for this patient?

1. Fibrosing mediastinitis

2. IgG4 - related sclerosing disease

3. Inflammatory myofibroblastic tumor

4. Lymphoma

5. Non-small cell carcinoma with a desmoplastic response 


\section{Correct! \\ 3. Inflammatory myofibroblastic tumor}

The surgical biopsy material, evaluated with immunohistochemistry and light microscopy, showed no evidence of malignancy, so both lymphoma and non-small cell carcinoma are excluded diagnoses. Immunoglobulin G4 (IgG4)-related sclerosing disease is a consideration, but is unlikely given the observation of the low number of IgG4 positive plasma cells in the biopsy material. Similarly, fibrosing mediastinitis is a prominent diagnostic consideration as well, but usually histopathologic material in patients with fibrosing mediastinitis shows less cellular and more fibrotic findings, which is not observed in this case. The most likely diagnosis is inflammatory myofibroblastic tumor.

Further clinical course: The patient was treated with corticosteroid therapy, with marked improvement in her systems. A repeat 18FDG-PET scan showed diminished metabolism within the right paramediastinal mass, and the mass decreased slightly in size as well.

Diagnosis: Inflammatory myofibroblastic tumor

\section{References}

1. Sugiyama K, Nakajima Y. Inflammatory myofibroblastic tumor in the mediastinum mimicking a malignant tumor. Diagn Interv Radiol. 2008; 14:197-199. [PubMed]

2. Agrons GA, Rosado-de-Christenson ML, Kirejczyk WM, Conran RM, Stocker JT. Pulmonary inflammatory pseudotumor: radiologic features. Radiology. 1998; 206:511-518. [CrossRef] [PubMed]

3. Coffin CM, Humphrey PA, Dehner LP. Extrapulmonary inflammatory myofibroblastic tumor: a clinical and pathological survey. Semin Diagn Pathol. 1998; 15:85-101. [PubMed]

4. Cerfolio RJ, Allen MS, Nascimento AG, Deschamps C, Trastek VF, Miller DL, Pairolero PC. Inflammatory pseudotumors of the lung. Ann Thorac Surg. 1999; 67:933-936. [CrossRef] [PubMed]

5. Kim TS, Han J, Kim GY, Lee KS, Kim H, Kim J. Pulmonary inflammatory pseudotumor (inflammatory myofibroblastic tumor): CT features with pathologic correlation. J Comput Assist Tomogr. 2005; 29:633-639. [CrossRef] [PubMed]

6. Chen $\mathrm{CH}$, Lin RL, Liu HC, Chen $\mathrm{CH}$, Hung TT, Huang WC. Inflammatory myofibroblastic tumor mimicking anterior mediastinal malignancy. Ann Thorac Surg. 2008; 86:1362-1364. [CrossRef] [PubMed]

7. Schaeffer CJ, Minai OA, Sharma N, Kanne JP, Mohammed TL. Inflammatory myofibroblastic tumor of the lung: recurrence after steroid treatment. J Thorac Imaging. 2008; 23:191-193. [CrossRef] [PubMed] 